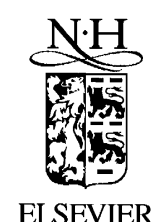

\title{
What have we learned from the kilowatt IR-FEL at Jefferson lab?
}

\author{
Stephen V. Benson* \\ Thomas Jefferson National Accelerator Facility, Laboratory MS 6a, 12000 Jefferson Avenue, Newport News, VA 23606, USA
}

\begin{abstract}
Recent work at Jefferson Lab has demonstrated the concept of same cell energy recovery to attain high average power in a free-electron laser. Since this device was the first of its kind, we learned a great deal about how to design such systems as we learned to operate the prototype. We are in the process of building a laser with an average power in excess of $10 \mathrm{~kW}$ in the infrared and have point designs for even higher power.

This work will summarize the problems which were thought to exist before the IR Demo lased and what we have learned since the laser operated successfully.

The upgrade has its own challenges and these will be described along with the proposed solutions. (C) 2002 Elsevier Science B.V. All rights reserved.
\end{abstract}

PACS: $29.20 . c ; 29.27 ; 41.60 . \mathrm{Cr} ; 42.60 . \mathrm{v}$

Keywords: High power; Mirror distortion; Power scaling

\section{Introduction}

The IR Demo free-electron laser (FEL) at Jefferson Lab has successfully operated with output power in excess of $2.1 \mathrm{~kW}$, and has energy recovered up to $180 \mathrm{~kW}$ of electron beam power [1]. Though using an old idea [2] and a conservative design, we were aware of many potentially fatal problems before building the system. Some examples of these problems are:

1. Poor quantum efficiency in GaAs photocathodes might make commissioning quite difficult due to changes in the beam properties with time.

\footnotetext{
*Tel.: + 1-757-269-5026; fax: + 1-757-269-5519.

E-mail address: felman@jlab.org (S.V. Benson).
}

2. No one had ever accelerated high average current from a DC photocathode gun. The potential for self-destruction or halo formation due to scattered light was a serious concern. Despite a very large energy spread, losses had to be limited to $0.1 \%$ total losses and $100 \mathrm{nA}$ at any point in the beam transport.

3. Though a numerical model indicated a threshold of $1.7 \mathrm{~mA}$ for the RF instability and the RF feedback system should have allowed operation well above that limit [3], it was feared that the model might not have included all physical effects.

4. Though the recirculation lattice used in the design had been demonstrated at MIT Bates [4], we know, from energy recovery on the Nuclear Physics injector that magnet quality must be 
excellent in the recirculation. The large energy spread must also be reduced very effectively to maintain acceptable beam quality at the dump. This meant canceling out the curvature in the RF waveform. The curvature alone caused a very large energy spread at the dump.

5. To save time and money, a simple nearconcentric resonator was chosen as the resonator for the IR Demo. It was known that mirror heating would distort the mirrors in such a way that the spot size on the mirrors would decrease, leasing to higher intensity and more distortion, ultimately limiting the laser power. Calculations showed that low-loss coatings on a high-conductivity substrate like sapphire should be able to operate at power levels in excess of $1 \mathrm{~kW}$ if the coating absorption were $<0.1 \%$ [5]. The possibility of some non-linear mechanism like a potato chip distortion or multi-phonon absorption might lead to power saturation at a much lower power level.

In the next section, I describe the resolution of these problems. In Section 3, I discuss the design of an upgrade capable of more than $10 \mathrm{~kW}$ of output power. In Section 4, I discuss the possibilities of even higher power.

\section{Lessons learned}

\subsection{Injector performance}

Early measurements showing a short lifetime for a GaAs DC photogun had the electron beam dump near the gun. With no beam loss in the vicinity of the gun, the lifetime turned out to be quite long. Recent measurements show a lifetime of $670 \mathrm{C}$ [6]. This allows many shifts of operation at full current before a cessation is required. One change compared to the original design is operating the laser spot off center on the cathode. The greatest ion back bombardment is in the center, so one can achieve extremely good lifetimes, if one operates a few millimeters offset from the cathode center. The cathode degradation mainly occurs at the operating spot and in the cathode center so one can further extend the operational lifetime by moving the spot [7].

The emittance, energy spread, and pulse length were not far from the values predicted by PARMELA. The original design assumed a bunch charge of $135 \mathrm{pC}$. Since the beam quality was quite good, we normally operate at $67 \mathrm{pC}$ or less. We have now measured the emittance at $135 \mathrm{pC}$. The injector emittance and energy spread are $25 \mathrm{~mm}$-mrad and $0.2 \% \mathrm{rms}$, respectively. This is good enough to provide for strong lasing with the high charge as well.

Though halo is created in the injector at low levels, the losses in the injector are quite small. Any halo that does exist tends to be scraped off only in the injector. The halo that makes it through the injection line is not lost in the rest of the high-energy transport. As a result, the beam losses at high energy are extremely low. Losses in any one point are much $<100 \mathrm{nA}$. The total measured losses at energies above $15 \mathrm{MeV}$ are typically much $<0.1 \%$. Operationally, this is extremely valuable since there is almost no beamline activation during operation even at the highest currents.

\subsection{Electron beam transport}

The Bates bends in the recirculation transport have proved to be a very robust optics solution. The design aperture is $5 \%$ but over $8 \%$ energy spread has been successfully energy recovered [8]. This has allowed operation with over $2.1 \mathrm{~kW}$ of continuous power. The limit to getting higher power from this record power is the available efficiency of the FEL ( $1 \%$ for this case). Higherorder dispersion in the lattice has not been a problem. The one unforeseen problem was the skew quadrupole focussing from the SRF cavities. Electromagnetic skew quadrupoles cancel out the effect of the RF skew quadrupole during acceleration but double the effect during deceleration. The net effect is to blow the recovered beam up vertically at the exit of the cryomodule and to create a skewed emittance at the dump.

The longitudinal performance of the lattice has also worked out quite well. The lattice is designed to rotate the synchrotron phase space by $90^{\circ}$ 
between the injector and the FEL. It then rotates the synchrotron phase space another $90^{\circ}$ between the FEL and the beam dump. Second-order correction is used to cancel out the curvature of the RF waveform so that the relative energy spread at the final dump is much smaller than the relative energy spread at the exit of the FEL. The FWHM energy spread at the dump is typically $<1 \%$ [9].

\section{3. $R F$ instabilities}

From the first recirculated lasing it was realized that the RF system was far more stable than we had hoped. Pulsed operation, even with quite short macropulses was quite easy to achieve. We now operate with macropulses as short as $6 \mu$ s (112 laser round trips). The high gain of the laser still permits saturation. The second observation was that operation near the peak of the detuning curve was actually quite stable. We were able to drive the system unstable near the peak of the detuning curve by adding a high-frequency energy oscillation [10], but the unperturbed system would not go unstable. We realized in hindsight that the $1.7 \mathrm{~mA}$ limit was not applicable to this design since there was basically no loss in the recirculation loop that might drive an instability [11]. The calculated open loop limit assuming only the phase instability is $27 \mathrm{~mA}$. With feedback the threshold is close to $1 \mathrm{~A}$. The system is therefore quite stable for just about any setup.

\subsection{Mirror distortion}

Though the FEL power is limited to $530 \mathrm{~W}$ with calcium fluoride mirrors, we have found that sapphire is quite capable of operating at well over $1 \mathrm{~kW}$ with no evidence of mirror heating. A model of the mirror distortion predicts that one should be able operate at up to $4 \mathrm{~kW}$ before the resonator mirrors limit the power [12]. With calcium fluoride mirrors, it is found that the efficiency peaks at $1.5 \mathrm{~mA}$ and about $350 \mathrm{~W}$. The power limit is about $50 \%$ higher. For sapphire mirrors the efficiency is still rising for the highest currents. This implies that the power limit is at least $3 \mathrm{~kW}$. We have not seen any evidence for non-linear absorption or potato chip distortion in the high-power mirrors.

\section{The IR upgrade design}

\subsection{Injector upgrade}

By upgrading the current, voltage, and laser efficiency, it is possible to scale the IR Demo to much higher power. The design specifications for such an upgrade are shown in Table 1. The electron beam energy is increased by adding two cryomodules, one the same as the present cryomodule and one including a seven-cell cavity with higher HOM capability. We will move the wiggler to the return leg. Calculations show that the emittance growth due to CSR is not a problem for

Table 1

Design parameters for the IR upgrade are compared with those of the IR demo and those achieved to date

\begin{tabular}{llll}
\hline & IR demo & Achieved & IR upgrade \\
\hline Energy (MeV) & $35-42$ & $20-48$ & $80-210$ \\
$I_{\text {avg }}(\mathrm{mA})$ & 5 & 5 & 10 \\
Beam power (kW) & 200 & 240 & 2000 \\
Charge/bunch (pC) & 135 & 135 & 135 \\
Repetition rate (MHz) & $18.75-37.43$ & $18.75-74.85$ & $4.68-74.85$ \\
FWHM bunch length (ps) & 2.5 & $1(60 \mathrm{pC})$ & 0.5 \\
Peak current (A) & 50 & $>60 \mathrm{~A}$ & 270 \\
$\Delta E / E$ (FWHM) & $<1.2 \%$ & $<1 \%$ & $<2 \%$ \\
$\varepsilon_{\mathrm{N}}(\mathrm{mm}-\mathrm{mrad})$ & 12 & $5-10(60 \mathrm{pC})$ & $<30$ \\
FEL efficiency (\%) & $0.6 \%$ & $>2 \%$ & $>1 \%$ \\
FEL power (kW) & 1 & 2.13 & $>10$ \\
Induced energy spread (\%) & $5 \%$ & $6-8 \%$ & $10 \%$ \\
\hline
\end{tabular}


this design [13]. The injector will be operated at twice the current of the IR Demo by operating at $135 \mathrm{pC}$ and $74.85 \mathrm{MHz}$. The beam quality required for the IR upgrade has already been demonstrated at $135 \mathrm{pC}$ and $37.425 \mathrm{MHz}$. The RF system and high-voltage power supply will be upgraded to handle the higher current and power required.

\subsection{Lattice upgrade}

The recirculation lattice has been described in detail elsewhere [13]. The bends at either end are still Bates style bends, but with a smaller maximum dispersion. Since we wanted to improve the efficiency, we also increased the energy acceptance up to $15 \%$. To make the synchrotron rotation uniform with such a large energy spread it was necessary to add third-order correction to the lattice after the FEL. Second-order correction before the FEL will allow compensation of the RF curvature and allow much shorter electron bunches than in the IR Demo (see Table 1). A chicane is used before the FEL to provide the final bunching and steer the electron beam around the upstream mirror. Larger beam tubes $(70 \mathrm{~mm}$ aperture) reduce the possibility of beam loss while simultaneously decreasing wakefields.

\subsection{Wiggler}

In order to provide the most flexibility and allow operation over a very large range of wavelengths, an optical klystron was chosen for the wiggler. This will allow the extraction efficiency to be varied continuously over a large range. The gain does not get extremely high in the klystron since the slippage becomes dominant for large dispersion. The optical klystron has two wigglers of 12 periods each separated by a dispersion section with 40 periods of path length delay at the highest wiggler strength. The wiggler period is $20 \mathrm{~cm}$ and the maximum rms $K^{2}$ is 16 . This allows the wavelength to be tuned over a very large range without resetting the accelerator energy. For example, at $130 \mathrm{MeV}$ one can tune from 2 to $25 \mu \mathrm{m}$ without changing the energy. Changing the relative strength of the two wigglers may allow slightly higher efficiency without larger exhaust energy spread [14].

\subsection{Resonator}

The power specification for the IR Demo was that the Rayleigh range must not increase by more than a factor of two. We have developed a technology which allows us to change the radius of curvature of a backplane cooled mirror while holding the figure to better than $\frac{1}{20}$ th of a wave [15]. We plan to use this type of mirror for the high reflector of the resonator. This means that it is possible to keep the Rayleigh range constant as the output coupler heats up. The power limit is now set by the induced aberration, waist movement, and the intensity on the mirrors.

In order to define these let us define a parameter $M \equiv 1+\left(L / 2 z_{\mathrm{R}}\right)^{2}$ Let us define a mean stability parameter $g$ and an offset variable $\delta$ so that

$$
\begin{aligned}
-\sqrt{g_{1} g_{2}} & \equiv g \\
\text { so } g_{1} & =g(1+\delta) \\
\text { and } g_{2} & =g /(1+\delta)
\end{aligned}
$$

where $g_{1}$ and $g_{2}$ are the usual cavity stability parameters. It is possible to show using the definition for $L / 2 z_{\mathrm{R}}$ [16] that

$$
M=\frac{1-\left(g_{1}+g_{2}\right)+\left(g_{1}+g_{2}\right)^{2} /\left(4 g^{2}\right)}{1-g^{2}} \text {. }
$$

Plugging Eq. (1) into Eq. (2) we find

$M \cong M_{\mathrm{s}}\left(1+\delta^{\prime}\right)$ where $\delta^{\prime}=\frac{\delta^{2}}{2(1+\delta)}$

and where $M_{\mathrm{s}} \equiv 2 /(1+g)$ is the magnification of a symmetric resonator with stability parameter $g$. The quantity $\delta^{\prime}$ is second order in the resonator asymmetry and so is quite small. It is reasonable to use the formulas for $M$ and $M_{\mathrm{S}}$ interchangeably.

From Ref. [16], the distance from the mode waist to the downstream mirror (defined here as mirror 1 ) is given by

$\frac{z_{1}}{L}=\frac{g_{2}\left(1-g_{1}\right)}{g_{1}+g_{2}-2 g_{1} g_{2}}$.

The offset of the optical waist from the cavity center can be found from substituting Eq. (1) into 
Eq. (4) and subtracting half the cavity length

$z_{\text {off }}=\frac{L}{2}\left(\frac{\delta^{\prime}-\delta}{1-g+\delta^{\prime}}\right) \cong-\frac{L}{2} \frac{\delta}{1-g}$.

Note that, if desired, one can start with a negative $z_{\text {off }}$ and move to a positive $z_{\text {off }}$ always staying close to the center. We would like the waist to stay within a certain fraction of a Rayleigh range of the center. This is true if

$\frac{z_{\text {off }}}{z_{r}} \cong \frac{L}{2 z_{r}} \frac{\delta}{1-g} \cong \frac{\delta}{2} \frac{M}{\sqrt{M-1}}<\left(\frac{\Delta z}{z_{\mathrm{R}}}\right)_{\mathrm{lim}}$.

If we assume that we start with a negative $\delta$ and move to positive $\delta$ as the mirror heats up and we assume that we keep the Rayleigh range constant by distorting the high reflector we find that [17]

$\frac{2 \delta g}{L} \cong \frac{1}{R_{\delta}}=\frac{c_{1} P_{1}}{8 M z_{\mathrm{R}} \lambda F}$

where

$F=\frac{k_{\mathrm{th}}}{\alpha_{\mathrm{e}}\left(h \alpha_{\mathrm{B}}+\alpha_{\mathrm{s}}+\alpha_{\mathrm{s}} / t_{\mathrm{c}}\right)}$

and $k_{\text {th }}$ is the thermal conductivity, $t_{\mathrm{c}}$ is the output coupler transmission, $\alpha_{B}$ is the bulk absorption/ $\mathrm{cm}, \alpha_{\mathrm{s}}$ is the coating absorption, $\alpha_{\mathrm{e}}$ is the expansion coefficient, and $h$ is the mirror thickness. This figure of merit depends explicitly only on measured properties of the mirror coatings and substrate. Since the bulk and surface absorption are wavelength dependent, the figure of merit will depend implicitly on the wavelength as well.

We can use Eqs. (6) and (7) to derive a limit for the power due to waist movement

$$
\begin{aligned}
P_{1} & <\frac{16(M-2)}{c_{1} M} \lambda F\left(\frac{\Delta z}{z_{\mathrm{R}}}\right)_{\lim } \\
& =\frac{13.7(M-2)}{M} \lambda F\left(\frac{\Delta z}{z_{\mathrm{R}}}\right)_{\mathrm{lim}} .
\end{aligned}
$$

We can also define power limitations from the aberrations in the resonator mirror figure and the allowed aberration in the beam transmitted through the output coupler [18]. They are

$$
\begin{aligned}
& P_{1}<41.2 \delta_{\mathrm{ab}} \lambda F \\
& \quad \text { and } P_{1}<\frac{20.6 \delta_{\mathrm{abt}}}{\left[(n-1)+1 / \alpha_{\mathrm{e}} \mathrm{d} n / \mathrm{d} T\right]} \lambda F
\end{aligned}
$$

where $\delta_{\mathrm{ab}}$ is the surface aberration limit per mirror and $\delta_{\mathrm{abt}}$ is the transmission aberration limit. If both mirrors are distorting $\delta_{\mathrm{ab}}$ is usually $\frac{1}{20}$. If only one mirror is distorting, it may be as large as $\frac{1}{10}$. The quantity $\delta_{\text {abt }}$ is usually $\frac{1}{5}$ but may be larger if aberration correction in the form of a deformable mirror is included in the optical transport system.

The power limit produced from an intensity limit on the mirrors is given by

$P_{1}<\frac{I_{\lim } M z_{\mathrm{R}} \lambda}{2 Q}$

where $I_{\mathrm{lim}}$ is the intensity limit on the mirror and $Q$ is the resonator $Q$. Since $M$ is inversely proportional to $z_{\mathrm{R}}^{2}$ for large $M$, one wants a small Rayleigh range, long cavity length, and a lowcavity $Q$ to get the highest power. This means that the magnification will get rather large.

One cannot make the magnification arbitrarily large when using a near-concentric resonator. For large $M$ and small $\delta$ the mode rotation and displacement for a mirror tilt $\theta_{\mathrm{m}}$ are given, to a good approximation, by $\theta \cong M_{\mathrm{s}} \theta_{\mathrm{m}}$ and $\Delta x \cong L \theta_{\mathrm{m}} / 2$. The magnification is thus limited by the engineering limit of how well the mirrors can be stabilized in angle. The limit is given by

$M<\frac{1}{6} \sqrt{\frac{\lambda}{\pi z_{\mathrm{R}}}} \frac{1}{\theta_{\mathrm{lim}}}$

where $\theta_{\lim }$ is the noise limit of the mirror angular feedback system.

The calculated limits for several different output coupler substrates and different wavelengths are shown in Table 2.

The lowest power limit at $1 \mu \mathrm{m}$ in Table 2 is due to the intensity at the mirror. With a different wiggler with a shorter Rayleigh range one could increase this by a factor of 10 before running into a limit of tolerable angular stability. At $3 \mu \mathrm{m}$ the ultimate power limit of $28.6 \mathrm{~kW}$ will be from surface aberration. Before that limit is reached, however, the output beam quality will degrade below desirable limits. This can, in principle, be corrected using a deformable mirror in the optical transport. At $6 \mu \mathrm{m}$ the ultimate power limit is only $16.6 \mathrm{~kW}$ but the output beam quality may be quite poor at that power. At $10 \mu \mathrm{m}$ the ultimate power limit is quite high at $38.7 \mathrm{~kW}$. The limit before 
Table 2

Power limits for some different resonator materials and wavelengths

\begin{tabular}{|c|c|c|c|c|c|}
\hline Substrate & Sapphire & Sapphire & $\mathrm{ZnSe}$ & $\mathrm{ZnSe}$ & $\mathrm{CaF}_{2}$ \\
\hline Wavelength $(\mu \mathrm{m})$ & 1 & 3 & 6 & 10 & 6 \\
\hline Bulk absorption (cm) & $0.05 \%$ & $0.1 \%$ & $0.1 \%$ & $0.05 \%$ & $0.05 \%$ \\
\hline Surface absorp. (ppm) & 50 & 100 & 100 & 100 & 100 \\
\hline Thickness (cm) & 0.625 & 0.625 & 1 & 1 & 1 \\
\hline $\mathrm{F}(\mathrm{kW} / \mu \mathrm{m})$ & 9.25 & 4.62 & 1.35 & 1.88 & 0.41 \\
\hline$P_{1}(\mathrm{~kW})$ change in $z$ & 24.5 & 36.8 & 21.4 & 49.8 & 6.5 \\
\hline$P_{1}(\mathrm{~kW})$ surf. aberr. & 19.0 & 28.6 & 16.6 & 38.7 & 5.0 \\
\hline$P_{1}(\mathrm{~kW})$ Intensity limit & 9.8 & 29.3 & 58.7 & 97.8 & 58.7 \\
\hline$P_{1}(\mathrm{~kW})$ trans. aberr. & 16.7 & 25.4 & 3.5 & 8.3 & 333.9 \\
\hline Angular stab. $(\mu \mathrm{rad})$ & 1.02 & 1.77 & 2.50 & 3.23 & 2.50 \\
\hline
\end{tabular}

Values in boldface are the ultimate power limits. Values in italics are lower limits due to output beam quality. The differences in $\mathrm{F}$ vs. wavelength for a given substrate are due to differences in the bulk and/or coating absorption.

output beam quality is poor, much lower but, at $8.3 \mathrm{~kW}$, is close to our goal of $10 \mathrm{~kW}$.

\section{Towards higher power}

By using a wiggler optimized for $1 \mu \mathrm{m}$ operation and installing two high gradient cryomodules it is possible to attain at least $20 \mathrm{~kW}$ at that wavelength in the IR upgrade. How can one go even higher? One path is to borrow a technique from the synchrotron radiation community and cool the silicon and sapphire mirrors to around $50 \mathrm{~K}$. At this temperature, the coefficient of thermal expansions for both materials become very small and the thermal conductivity of sapphire increases dramatically from the room temperature value [19]. This can increase the value of $F$ by over a factor of 200 . The power limits from aberration now are measured in megawatts. The limiting factor would now be the intensity on the mirrors. Assuming a noise level of $100 \mathrm{nrad}$ for mirror stability is attainable with cooled mirrors and that the intensity should be limited to $100 \mathrm{~kW} / \mathrm{cm}^{2}$, one can show that the power at $1 \mu \mathrm{m}$ is limited to $100 \mathrm{~kW}$ for $15 \%$ output coupler transmission. Increasing the gain and lower the resonator $Q$ might raise this further. If the efficiency can be raised to $2.5 \%$ in such a device, the IR upgrade could achieve $50 \mathrm{~kW}$ with only $10 \mathrm{~mA}$ of beam current. If the beam current were increased to
$50 \mathrm{~mA}$ and the efficiency remained at $1 \%$, one could achieve $100 \mathrm{~kW}$.

\section{Conclusions}

We have learned a great deal about energy recovering Linac-based FELs since we first designed the IR Demo FEL. Many of the issues that were feared to limit the operation of such devices are now understood. The road ahead looks much more open and evolution of these devices towards over $100 \mathrm{~kW}$ looks quite reasonable.

\section{Acknowledgements}

Many thanks to all the technical staff at Jefferson Lab who helped make this work possible. This work was supported by the US Department of Energy under Contract DE-AC0584-ER40150, the Office of Naval Research, the Commonwealth of Virginia, and the Laser Processing Consortium.

\section{References}

[1] G.R. Neil, et al., Phys. Rev. Lett. 84 (2000) 662.

[2] M. Tigner, NuovoCimento 37 (1965) 1228.

[3] L. Merminga, P. Alexeev, S. Benson, A. Bolshakov, L. Doolittle, G. Neil, Nucl. Instr. and Meth. A 429 (1999) 58. 
[4] J.B. Flanz, C.P. Sargent, Nucl. Instr. and Meth. A 241 (1985) 325.

[5] S.V. Benson, P.S. Davidson, R. Jain, P.K. Kloeppel, G.R. Neil, M.D. Shinn, Nucl. Instr. and Meth. A 407 (1998) 401.

[6] T. Siggins, et al., Performance of a DC GaAs photocathode gun for the Jefferson Lab FEL, Nucl. Instr. and Meth. A 475 (2001) 549.

[7] C.K. Sinclair, Recent advances in polarized electron sources, Proceedings PAC '99, New York, NY, p. 65.

[8] S. Benson, J. Gubeli, G.R. Neil, An experimental study of an FEL oscillator with a linear taper, Nucl. Instr. and Meth. A 475 (2001) 276.

[9] The Jefferson Lab $1 \mathrm{~kW}$ IRFEL, Presented at the Linac 2000 Conference, Aug. 2000, Monterey, CA, http:// www.slac.stanford.edu/econf/C000821/TH204.pdf.

[10] L. Merminga, First experimental data on the FEL-RF interaction at the Jefferson Lab IRFEL, in J. Feldhaus, H. Weise, Proc. of FEL 1999. Elsevier Science B.V., Amsterdam, 2000, p. II-3.

[11] L. Merminga, RF stability in energy recovering free electron lasers: theory and experiment, Nucl. Instr. and Meth. A 483 (2002), these proceedings.

[12] S.V. Benson, G.R. Neil, M. Shinn, Measurement and modeling of mirror distortion in a high power FEL, in: $\mathrm{S}$.
Basu, S. J. Davis, E. A. Dorko (Eds.), Gas, Chemical, and Electrical Lasers and Intense Beam Control and Applications; Proc. SPIE 3931 (2000) 243-250.

[13] D. Douglas, S. V. Benson, G. A. Krafft, R. Li, L. Merminga, B. C. Yunn, Driver accelerator design for the $10 \mathrm{~kW}$ upgrade of the Jefferson Lab IRFEL D, Presented at the Linac 2000 Conference, Monterey, CA, August 2000 .

[14] D.A. Jaroszynski, et al., Nucl. Instr. and Meth. A 358 (1995) 228.

[15] Patent application submitted.

[16] A.E. Siegman, Lasers, University Science Books, Mill Valley, CA, 1986.

[17] G.R. Neil, S.V. Benson, M.D. Shinn, P.S. Davidson, P.K. Kloeppel, Optical modeling of the Jefferson laboratory IR demo FEL, Modeling and Simulation of Higher Power Laser Systems IV; Proc. SPIE 2989 (1997) 160-171.

[18] S. Benson, M. Shinn, Focusing effects due to mirror heating in the IR demo optical resonator, Jefferson Lab Technical Note JLAB-TN-98-015.

[19] R.J. Corruccini, J.J. Gniewek, Thermal expansion of technical solids at low temperatures, a compilation from the literature, National Bureau of Standards Monograph, Vol. 29, 1961. 